\title{
Likelihood ratio testing for hidden Markov models under nonstandard conditions ${ }^{1}$
}

\author{
JÖRN DANNEMANN \\ Institut für Mathematische Stochastik, University of Göttingen \\ HAJO HOLZMANN ${ }^{2}$ \\ Institut für Stochastik, University of Karlsruhe
}

\begin{abstract}
In practical applications, when testing parametric restrictions for hidden Markov models (HMMs) one frequently encounters non-standard situations such as testing for zeroentries in the transition matrix, one-sided tests for the parameters of the transition matrix or for the components of the stationary distribution of the underlying Markov chain, or testing boundary restrictions on the parameters of the state-dependent distributions. In this paper we briefly discuss how the relevant asymptotic distribution theory for the likelihood ratio test (LRT) when the true parameter is on the boundary extends from the i.i.d. situation to HMMs. Then we concentrate on discussing a number of relevant examples. The finite-sample performance of the LRT in such situations is investigated in a simulation study. An application to series of epileptic seizure counts concludes the paper.
\end{abstract}

Key words: bimodality, boundary, hidden Markov model, likelihood ratio test, marginal distribution, maximum likelihood estimation, overdispersion

\section{Introduction}

Hidden Markov models (HMM) are a class of discrete-time stochastic processes that have been successfully applied in various fields of dependent data analysis, including speech recognition (Rabiner, 1989), econometrics (Rydén et al., 1998), biology (Holzmann et al., 2006), medical statistics (Albert, 1991) or biological sequence alignment (Arribas-Gil et al., 2006). See the monograph by MacDonald \& Zucchini (1997) for further examples.

An HMM consists of two ingredients, an unobservable finite-state Markov chain $\left(X_{k}\right)$ and an observable stochastic process $\left(Y_{k}\right)$ such that (i) the $\left(Y_{k}\right)$ are conditionally independent, given the $\left(X_{k}\right)$ and (ii) given the $\left(X_{k}\right)$, the distribution of $Y_{j}$ depends on $X_{j}$ only. Since the Markov chain $\left(X_{k}\right)$, which is sometimes called the regime or the latent process, is unobservable, inference has to be based on the $\left(Y_{k}\right)$ alone. Typically it is assumed that the state dependent

\footnotetext{
${ }^{1}$ Preprint submitted to the Scandinavian Journal of Statistics (25 Oct 2007).

${ }^{2}$ Corresponding author: holzmann@stoch.uni-karlsruhe.de
} 
distributions, i.e. the distributions of $Y_{k}$ given that $X_{k}=a, a \in \mathcal{M}$, come from a parametric family $\left(f_{\theta}\right)_{\theta \in \Phi}$ of densities or discrete distributions, e.g. the normal or the Poisson distribution. Thus, the unknown parameters in an HMM involve both the transition probabilities of the Markov chain and the parameters of the state dependent distributions. The major approach to estimate the parameters in an HMM is via likelihood-based methods. For general HMMs, strong consistency of the maximum likelihood estimator (MLE) was proved by Leroux (1992). Bickel et al. (1998) established asymptotic normality of the score with limit covariance matrix $\mathcal{J}_{0}$, as well as a uniform law of large numbers for the Hessian of the log-likelihood with limit matrix $-\mathcal{J}_{0}$ (for related results see also Douc et al., 2004). Once these major results are obtained, the standard likelihood theory such as asymptotic normality of the MLE with limit covariance $\mathcal{J}_{0}^{-1}$ (cf. Bickel et al., 1998) and the asymptotic $\chi^{2}$-approximation to the distribution of the likelihood-ratio test (LRT) under regularity conditions (cf. Giudici et al., 2000) follows as in the i.i.d. setting.

In this paper we observe that also the likelihood theory under non-standard conditions with parameters on the boundary, as developed by Chernoff (1954) and Self \& Liang (1987), can be extended from the i.i.d. case to HMMs by using the results of Bickel et al. (1998). In particular, we give the asymptotic distribution theory for the LRT for general, nonlinear hypotheses with parameters on the boundary, and these parameters might also involve the parameters of the state-dependent distributions.

Indeed, such testing situations are frequently encountered in practice when using HMMs. For example, if one wishes to test whether the state $a$ is always left immediately, or whether the underlying Markov chain tends to stay in the state $a$, or whether the state $a$ is on average more frequently visited than the state $b$, one requires testing for zero-entries of the transition matrix, testing a one-sided hypothesis on the parameters of the transition matrix and on the parameters of the stationary distribution of the underlying Markov chain, respectively. Hence,

all these testing problems and several others involve the extended asymptotic distribution of the LRT with true parameter on the boundary of the hypothesis.

The paper is organized as follows. In Section 2, after formally introducing HMMs, we briefly discuss how the asymptotic distribution theory for the LRT under non-standard conditions can be extended from the i.i.d. case to HMMs. An extensive list of examples is given in Section 3. In Section 4 we present simulation results and illustrate the application of the tests for a series of epileptic seizure count data, previously analyzed by Le et al. (1992). Some proofs are given in an appendix.

\section{Likelihood inference for HMMs}

Let us start by introducing some further notation and definitions. Let $\left(X_{k}\right)_{k \geq 1}$ be a stationary, finite-state Markov chain with state space $\mathcal{M}=\{1, \ldots, m\}$, transition probabilities $\alpha_{a b}=P\left(X_{k+1}=b \mid X_{k}=a\right)$, and unique stationary distribution $\pi=\left(\pi_{1}, \ldots, \pi_{m}\right)$. Further 
let $\left(Y_{k}\right)_{k \geq 1}$ be a stochastic process taking values in a Borel-measurable subset $\mathcal{Y}$ of Euclidean space, such that given $\left(X_{k}\right)_{k \geq 1}$, the $\left(Y_{k}\right)_{k \geq 1}$ are independent and the distribution of $Y_{k}$ depends on $X_{k}$ only. These conditional distributions are called the state-dependent distributions and we assume that they come from a parametric family $\{f(y ; \theta) \mid \theta \in \Phi\}$ of densities w.r.t. a $\sigma$-finite measure $\nu$ on $\mathcal{Y}$, so that the distribution of $Y_{k}$, given that $X_{k}=a$, has density $f\left(\cdot ; \theta_{a}\right)$. We assume that both the parameters of the transition matrix $\left\{\alpha_{a b}\right\}=\left\{\alpha_{a b}(\vartheta)\right\}$ and the parameters of the state-dependent densities $\theta_{a}=\theta_{a}(\vartheta)$ depend on a parameter $\vartheta \in \Theta \subset \mathbb{R}^{d}$. The standard parametrization is given by $\vartheta=\left(\alpha_{11}, \ldots, \alpha_{1, m-1}, \alpha_{21}, \ldots, \alpha_{m, m-1}, \theta_{1}, \ldots, \theta_{m}\right)$. The subindex 0 indicates the true value $\vartheta_{0}$ and the true distribution $P_{0}$ of the bivariate process $\left(X_{k}, Y_{k}\right)_{k \geq 1}$. Note that since the parameters of the transition matrix $\alpha_{a b}(\vartheta)$ depend on $\vartheta$, so do the components of the unique stationary distribution $\pi_{a}=\pi_{a}(\vartheta)$.

The joint density of $\left(X_{1}, \ldots, X_{n}, Y_{1}, \ldots, Y_{n}\right)$ (w.r.t. (counting measure) $\left.{ }^{n} \times \nu^{n}\right)$ is given by

$$
p_{n}\left(x_{1}, \ldots, x_{n}, y_{1}, \ldots, y_{n} ; \vartheta\right)=\pi_{x_{1}}(\vartheta) \prod_{k=1}^{n-1} \alpha_{x_{k}, x_{k+1}}(\vartheta) \prod_{k=1}^{n} f\left(y_{k} ; \theta_{x_{k}}(\vartheta)\right),
$$

the joint density of $\left(Y_{1}, \ldots, Y_{n}\right)$ (w.r.t. $\left.\nu^{n}\right)$ by

$$
p_{n}\left(y_{1}, \ldots, y_{n} ; \vartheta\right)=\sum_{x_{1}=1}^{m} \cdots \sum_{x_{n}=1}^{m} p_{n}\left(x_{1}, \ldots, x_{n}, y_{1}, \ldots, y_{n} ; \vartheta\right),
$$

and the $\log$ likelihood is denoted by $L_{n}(\vartheta)=\log p_{n}\left(y_{1}, \ldots, y_{n} ; \vartheta\right)$. A maximum likelihood estimator (MLE) $\hat{\vartheta}$ is any value of $\vartheta \in \Theta$ which maximizes $L_{n}(\vartheta)$.

Leroux (1992) gives conditions for HMMs with finite state space and general observational space $\mathcal{Y}$ under which the MLE is strongly consistent, i.e. $\hat{\vartheta} \rightarrow \vartheta_{0} P_{0}$-a.s. as $n \rightarrow \infty$. Denote the matrix of second derivatives of $L_{n}(\vartheta)$ by $D_{\vartheta}^{2} L_{n}(\vartheta)$. Bickel et al. (1998) show that if $\tilde{\vartheta}_{n}$ is any strongly consistent sequence of estimates, under their Assumptions 1-4,

$$
n^{-1} D_{\vartheta}^{2} L_{n}\left(\tilde{\vartheta}_{n}\right) \rightarrow-\mathcal{J}_{0} \quad \text { in } P_{0} \text {-probability, }
$$

as $n \rightarrow \infty$. The matrix $\mathcal{J}_{0}$ is called the Fisher information matrix. Douc et al. (2004) extend (1) to almost sure convergence. Bickel et al. (1998) further show under their Assumptions 1-4 a central limit theorem for the score, $\sqrt{n} D_{\vartheta} L_{n}\left(\vartheta_{0}\right) \stackrel{\mathcal{L}}{\rightarrow} N\left(0, \mathcal{J}_{0}\right)$.

Once these fundamental results are established, the standard likelihood theory can be derived in complete analogy to the i.i.d. case. Bickel et al. (1998) conclude that if $\vartheta_{0}$ is an interior point of $\Theta$ and if $\mathcal{J}_{0}$ is nonsingular, $\sqrt{n}\left(\hat{\vartheta}-\vartheta_{0}\right)$ is asymptotically normally distributed with mean zero and covariance matrix $\mathcal{J}_{0}^{-1}$. Giudici et al. (2000) extend the $\chi^{2}$-approximation for the LRT for regular hypotheses from the i.i.d. setting to HMMs.

However, we observe that this strategy also applies when treating the LRT in case the true parameter is on the boundary of the hypothesis, cf. Chernoff (1954) and Self \& Liang (1987) for the i.i.d. setting. To formulate the results, for $\Theta_{0} \subset \Theta$ let

$$
\lambda_{n}=\frac{\sup _{\vartheta \in \Theta_{0}} p_{n}\left(y_{1}, \ldots, y_{n} ; \vartheta\right)}{\sup _{\vartheta \in \Theta} p_{n}\left(y_{1}, \ldots, y_{n} ; \vartheta\right)}
$$


be the likelihood ratio. If Assumptions 1-4 in Bickel et al. (1998) hold true, if both $\Theta$ and $\Theta_{0}$ satisfy Assumption 1 (cf. the Appendix), if $\mathcal{J}_{0}$ is nonsingular and if $\Theta$ can be approximated by a cone $C_{\Theta}$ at $\vartheta_{0}$, and if $\Theta_{0}$ can be approximated by a cone $C_{\Theta_{0}}$ at $\vartheta_{0}$ (cf. the Appendix for the definition), then

$$
T_{n}=-2 \log \lambda_{n} \stackrel{\mathcal{L}}{\rightarrow} \min _{z \in C_{\Theta_{0}}-\vartheta_{0}}(Z-z)^{T} \mathcal{J}_{0}(Z-z)-\min _{z \in C_{\Theta}-\vartheta_{0}}(Z-z)^{T} \mathcal{J}_{0}(Z-z),
$$

where $Z \sim N\left(0, \mathcal{J}_{0}^{-1}\right)$, and $\mathcal{J}_{0}$ is the Fisher information matrix in an HMM as given in (1). The asymptotic distribution in (2) in general depends on the Fisher information matrix. In some examples (cf. Example 3 in the next section), it can be evaluated algebraically, otherwise, if required it has to be estimated, for example, by using a version of the forward algorithm for computing the observed information matrix (cf. Lystig \& Hughes, 2002). Alternatively, if one uses direct numerical maximization for computation of the MLE $\hat{\vartheta}$, most algorithms also give an estimate of the Hessian matrix at $\hat{\vartheta}$. From (1) one can then in principle determine an estimate of $\mathcal{J}_{0}$. Still, estimation of $\mathcal{J}_{0}$ is a difficult problem, and the approximation (2) works best if the right side does not depend on $\mathcal{J}_{0}$.

\section{$3 \quad$ Examples}

Example 1 (Zero entries of the transition matrix). In order to reduce the number of parameters in an HMM, and sometimes also from the context of the statistical problem, it is reasonable to restrict attention to transition matrices with certain prespecified zero entries (for an example see (9)). Therefore, testing for zeros in the transition matrix is evidently of some practical interest. Such boundary cases for LR testing were also studied by Bartolucci (2006) for latent Markov models, and here we briefly discuss them in a general HMM framework. Consider the hypothesis that a certain entry $\alpha_{a b}$ of the transition matrix is zero:

$$
H_{a b}^{0}: \quad \alpha_{a b}=0 \quad \text { against } \quad K_{a b}^{0}: \quad \alpha_{a b}>0,
$$

where it is assumed that under $H_{a b}^{0}$, the transition matrix still is ergodic, and the parameters of the state-dependent distributions are allowed to vary. In case of two states, if $\alpha_{21} \neq 0$ and $\alpha_{22} \neq 0$ this is true for $\alpha_{11}=0$ but evidently not for $\alpha_{12}=0$. Under $H_{a b}^{0}$, from case 5 in Self \& Liang (1987) it follows that

$$
T_{n} \stackrel{\mathcal{L}}{\rightarrow} \frac{1}{2} \chi_{0}^{2}+\frac{1}{2} \chi_{1}^{2},
$$

where $\chi_{0}^{2}$ is the point measure at 0 . If one combines several of the $H_{a b}^{0}$-type hypotheses, $T_{n}$ will have a $\bar{\chi}^{2}$-distribution (Bartolucci, 2006), where the weights in the $\bar{\chi}^{2}$-distribution can be determined from the entries of the Fisher information matrix (cf. Silvapulle \& Sen, 2005, for a simulation procedure). Thus, a joint test would involve estimation of the Fisher information matrix. A simpler, though less efficient method would be to test several of the $H_{a b}^{0}$-type hypotheses via some multiple testing procedure. 
Example 2 (Boundary cases for parameters of the state-dependent distributions). Another possibility for model reduction is to test whether certain parameters of the state-dependent distributions are on the boundary of their parameter spaces.

As a first example, suppose that the state-dependent distributions are Bernoulli $B(p)$, and that the underlying Markov chain has two states. MacDonald \& Zucchini (1997, pp. 140-144) consider the situation that in one of the states of the Markov chain (e.g. state 1) the outcome is just deterministic. This can be formulated by testing $H: p_{1}=0$ against $K: p_{1}>0$. The corresponding LRT again has the asymptotic distribution (3).

Another example arises in the analysis of count data with overdispersion relative to the Poisson distribution (Leroux \& Puterman, 1992). Such data is often modeled by finite mixtures of Poisson distributions, and HMMs then provide a natural generalization to the time-series context. Often, overdispersion mainly arises since there are too many zero-observations (relative to Poisson), which is then modeled by zero-inflation of the Poisson distribution (van den Broek, 1995). This can be interpreted as a two-component Poisson mixture, where $\lambda=0$ for one of the components. Thus, in the context of overdispersed count series, testing for zero inflation against general overdispersion structure can be accomplished by testing $H: \lambda_{1}=0$ against $K: \lambda_{1}>0$, with two-state underlying Markov chain and $\lambda_{1}<\lambda_{2}$. The LRT again has the asymptotic distribution (3).

Example 3 (One-sided tests for the transition probabilities). In this example we consider one-sided hypotheses for the entries of the transition matrix. First consider

$$
H: \alpha_{a c} \geq \alpha_{b c} \quad \text { against } \quad K: \alpha_{a c}<\alpha_{b c}
$$

where $a, b, c \in \mathcal{M}$, i.e. that it is more probable under the alternative to have reached state $c$ coming from state $b$ than coming from state $a$. On the boundary of $H$, i.e. for $\alpha_{a c}=\alpha_{b c}$, $T_{n}$ has the asymptotic distribution (3), and if $\alpha_{a c}>\alpha_{b c}$, by strong consistency of the MLE, $T_{n} \rightarrow 0$ in probability. Similarly one can test $H: \alpha_{a b} \geq \alpha_{a c}$ against $K: \alpha_{a b}<\alpha_{a c}$, that under the alternative it is more likely to go from $a$ to $c$ than to $b$, or $H_{a b}^{q}: \alpha_{a b} \leq q$ against $K_{a b}^{q}: \alpha_{a b}>q$ for some $q \in(0,1)$ and $a, b \in \mathcal{M}$.

For the $H_{a b}^{q}$-type hypothesis, a relevant special case is when $a=b$ and $q=1 / 2$, since in this case, under the alternative the HMM tends to stay in state $a \in \mathcal{M}$. We shall call such a state stable. Let us consider joint tests on two states $a, b \in \mathcal{M}$. First consider the testing problem

$$
H_{a \wedge b, 1 / 2}: \quad \alpha_{a a}=1 / 2 \wedge \alpha_{b b}=1 / 2 \quad \text { against } \quad K_{a \wedge b}: \quad \alpha_{a a}>1 / 2 \wedge \alpha_{b b}>1 / 2 .
$$

We shall only derive the limit law in a special situation, namely if there are only two states and only the transition probabilities are allowed to vary. Using case 7 in Self \& Liang (1987), one shows that under $H_{a \wedge b, 1 / 2}$,

$$
T_{n} \stackrel{\mathcal{L}}{\rightarrow}\left(\frac{1}{2}-p\right) \chi_{0}^{2}+\frac{1}{2} \chi_{1}^{2}+p \chi_{2}^{2},
$$


where the mixing quantity $p$ can be evaluated as

$$
p=\left(\cos ^{-1} \rho\right) /(2 \pi)
$$

with $\rho$ given in (12) in the appendix, which also contains the proof.

The testing problem (4) is somewhat artificial, and if one intends to test the hypothesis that neither state is stable against the alternative that both states are stable, the testing problem should be formulated as

$$
H_{a \wedge b}: \quad \alpha_{a a} \leq 1 / 2 \wedge \alpha_{b b} \leq 1 / 2 \quad \text { against } \quad K_{a \wedge b}: \quad \alpha_{a a}>1 / 2 \wedge \alpha_{b b}>1 / 2 .
$$

It turns out that in this testing problem, the asymptotic distribution given in (2) is no longer a finite mixture of $\chi^{2}$-distributions with different degrees of freedom (cf. Self \& Liang, 1987, for an example where a nuisance parameter is on the boundary of the hypothesis). Here the reason is that the whole parameter space under investigation, $\Theta=K_{a \wedge b} \cup H_{a \wedge b}$ is not convex. The asymptotic distribution of $T_{n}$ for the testing problem (7) in case of two states with known state-dependent distributions for $\alpha_{a a}=\alpha_{b b}=1 / 2$ turns out to be

$$
T_{n} \stackrel{\mathcal{L}}{\rightarrow} \frac{1}{2} \chi_{0}^{2}+\frac{\pi-\phi}{2 \pi} \chi_{2}^{2}+\frac{\pi-\phi}{2 \pi} P_{1}(\phi)+\frac{2 \phi-\pi}{2 \pi} P_{2}(\phi),
$$

where $\phi=\cos ^{-1} \rho \in[\pi / 2, \pi), \rho$ is given in (12) and the densities of $P_{1}(\phi)$ and $P_{2}(\phi)$ are specified in (14) and (15) in the appendix, which also contains the proof. For other parameter constellations under $H_{a \wedge b}$, the limit distribution is stochastically smaller. The asymptotic distributions (5) and (8) of the closely related testing problems (4) and (7) differ surprisingly strongly. Since testing problems which lead to these asymptotics arise in other contexts as well, this example is of more general interest, also for the i.i.d. setting.

Example 4 (Tests on the stationary distribution). For an ergodic Markov chain, the transition probability matrix uniquely determines the stationary distribution $\pi$. Hence, tests on the entries of $\pi$ can be reformulated into tests for the entries of the transition probability matrix, and (2) in principle allows to test one-sided hypotheses such as $\pi_{a} \geq \pi_{b}$ for $a, b \in \mathcal{M}$. However, the formulas for $\pi$ in terms of the $\alpha_{a b}$ are highly non-linear for more than two states, which makes explicit maximization under the hypothesis difficult. We illustrate this issue for two states and for a certain type of transition matrices in case of three states.

First consider the case of two states, and suppose that we want to test certain restrictions on $\pi_{1}$ (or equivalently on $\pi_{2}=1-\pi_{1}$ ), where the state-dependent parameters are allowed to vary. Let $\alpha_{12}=\alpha$ and $\alpha_{21}=\beta$, then $\pi_{1}=\beta /(\alpha+\beta)$. Consider testing the hypothesis that state 1 is on average at least as often visited as state 2, i.e. $H: \pi_{1} \geq \pi_{2}$ against $K: \pi_{1}<\pi_{2}$. Evidently, $H$ is equivalent to the linear restriction $\beta \geq \alpha$, and on the boundary of $H$, i.e. for $\alpha=\beta \neq 1$, one has the asymptotic distribution given in (3). Similarly, general restrictions $H_{p}: \pi_{1} \leq p$ for some $p \in(0,1)$ can be formulated into linear restrictions $H_{p}:(1-p) \beta \leq p \alpha$, 
and on the boundary of the hypothesis, (3) applies as well.

Next we consider HMMs with three states, where the transition matrix is supposed to be given by

$$
\left(\begin{array}{ccc}
1-\alpha & \alpha & 0 \\
\beta & 1-\beta-\gamma & \gamma \\
0 & \delta & 1-\delta
\end{array}\right)
$$

Here state 2 can be interpreted as a transitory state, through which every transition from state 1 to state 3 has to pass. The stationary distribution is given by

$$
\pi_{1}=\frac{\delta \beta}{\delta \beta+\delta \alpha+\gamma \alpha}, \quad \pi_{2}=\frac{\delta \alpha}{\delta \beta+\delta \alpha+\gamma \alpha}, \quad \pi_{3}=\frac{\gamma \alpha}{\delta \beta+\delta \alpha+\gamma \alpha} .
$$

Evidently, even linear restrictions on the parameters $\pi_{1}$ will lead to nonlinear restrictions for the parameters of the transition matrix. For example, consider the hypothesis $H_{1,3}: \pi_{1} \geq \pi_{3}$, which is equivalent to $H_{1,3}: \delta \beta \geq \gamma \alpha$, and one again obtains the asymptotic (3) for $T_{n}$. Further, if one wishes to test $H_{1,2,3}: \pi_{1}=\pi_{3}, \pi_{1}>\pi_{2}$, which is equivalent to $H_{1,2,3}: \delta \beta=$ $\gamma \alpha, \beta>\alpha$, then $T_{n} \stackrel{\mathcal{L}}{\rightarrow} \frac{1}{2} \chi_{1}^{2}+\frac{1}{2} \chi_{2}^{2}$ (cf. case 6 in Self \& Liang, 1987)

Example 5 (Testing for bimodality). Suppose that we have a two-state Gaussian HMM. Then the marginal distribution will be a two-component mixture of normals, where the mixing proportions are given by $\pi_{1}=\beta /(\alpha+\beta)$ and $\pi_{2}=1-\pi_{1}$, using the notation in Example 4 . A two component mixture of normals is either unimodal or bimodal, and explicit characterizations for unimodal parameter regions are available (Robertson \& Fryer, 1969). For example, for $\pi_{1}=1 / 2$, the mixture is unimodal if and only if $\left|\mu_{1}-\mu_{2}\right| \leq 2 \sigma$. Using these facts, one can construct a LRT for bimodality of the marginal distribution of a two-state normal HMM by using Theorem 1. To this end, the model has to reparametrized in $\left(\pi_{1}, \beta\right)$ instead of $(\alpha, \beta)$, and the restriction of unimodality can then be formulated in terms of $\pi_{1}$ and the parameters of the state dependent distributions. Let $\Theta_{0}$ be the unimodal parameter region. Then for all $\vartheta \in \Theta_{0}$, the asymptotic distribution of $T_{n}$ is stochastically smaller than $\left(\chi_{0}^{2}+\chi_{1}^{2}\right) / 2$, and for some parameter values on the boundary of $\Theta_{0}$ there is equality. Therefore, a test based on the critical value of $\left(\chi_{0}^{2}+\chi_{1}^{2}\right) / 2$ asymptotically keeps the level for all $\vartheta \in \Theta_{0}$. The test can be extended to other families of state-dependent distributions for which a characterization of the modality in two-component mixtures is available (e.g. the von Mises distribution, cf. Mardia \& Sutton, 1975, or the multivariate normal distribution, cf. Ray \& Lindsay, 2005).

\section{Simulations and empirical illustration}

\subsection{Quality of asymptotic approximation for the LRT}

As advocated by MacDonald \& Zucchini (1997), for the numerical computation of the maximum likelihood estimates we use direct maximization via a Newton-type algorithm. In each 
setting we reparametrize the problem so that unconstrained maximization is possible.

a. Consider testing the hypothesis $H: \alpha_{11}=0$ in a stationary two-state normal HMM, as described in Example 1, where the asymptotic distribution for $T_{n}$ is given in (3). The transition matrix is taken as

$$
A=\left(\begin{array}{cc}
0 & 1 \\
0.3 & 0.7
\end{array}\right) \text {, }
$$

and for the parameters of the state-dependent distributions we choose $\sigma^{2}=1$ and mean values $\mu_{1}=0, \mu_{2}=2$ in the first setting, which corresponds to sufficiently well-separated state-dependent distributions, and $\mu_{1}=0, \mu_{2}=1$ in the second setting, where the statedependent distributions strongly overlap. In the simulation we fix the parameters of the state-dependent distributions at their true values, and let only the parameters of the transition matrix vary. We generate $N=10000$ samples of various sizes, and for visualization we use PP-plots, which show for each nominal level $1-\alpha$ the empirical probability that the LRT statistic $T_{n} \leq q_{1-\alpha}$, where $q_{1-\alpha}$ is the $1-\alpha$-quantile of the asymptotic distribution.

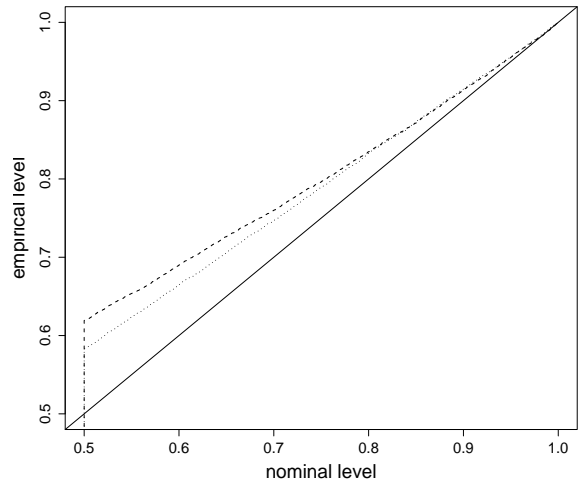

Figure 1: PP Plot of distribution of $T_{n}$ for $n=100$ (dashed line) and $n=500$ (dotted line) for hypothesis $H: \alpha_{11}=0$ in case $\mu_{1}=0, \mu_{2}=2$.

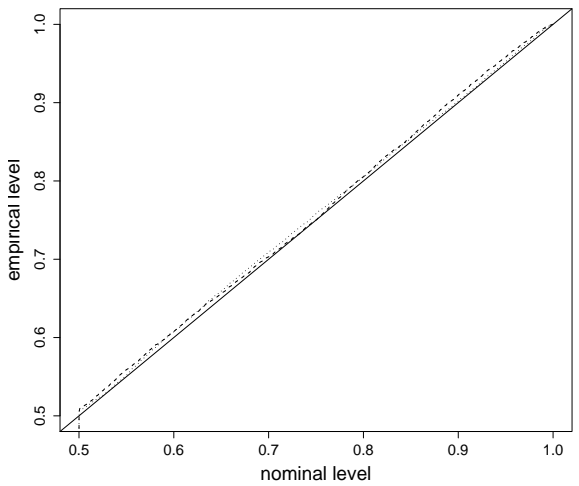

Figure 2: PP Plot of distribution of $T_{n}$ for $n=50$ (dashed line) and $n=100$ (dotted line) for hypothesis $H: \alpha_{11}=0$ in case $\mu_{1}=0, \mu_{2}=1$.

The results are displayed in Figs. 1 and 2. It turns out that the asymptotic approximation for well separated state-dependent distributions is relatively poor, even for large sample sizes such as $n=500$ and in this simple situation with fixed parameters for the state-dependent distributions, while for strongly overlapping state-dependent distributions the approximation is quite good already for $n=50$.

b. Further, we examine testing the hypothesis $H_{1,3}: \pi_{1} \geq \pi_{3}$ as described in Example 4 for a stationary three-state Poisson HMM. We use a transition matrix as in (9), with $\alpha=0.4$, $\beta=0.2, \gamma=0.3$ and $\delta=0.6$, yielding for the state-dependent distribution $\pi_{1}=\pi_{3}=0.25$, 


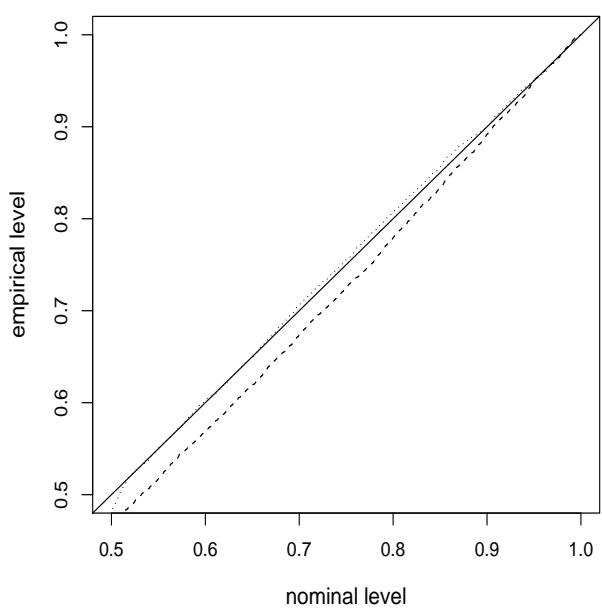

Figure 3: PP Plot of distribution of $T_{n}$ for $n=100$ (dashed line) and $n=500$ (dotted line) for hypothesis $H_{1,3}$ in case of fixed and known $\lambda$ 's.

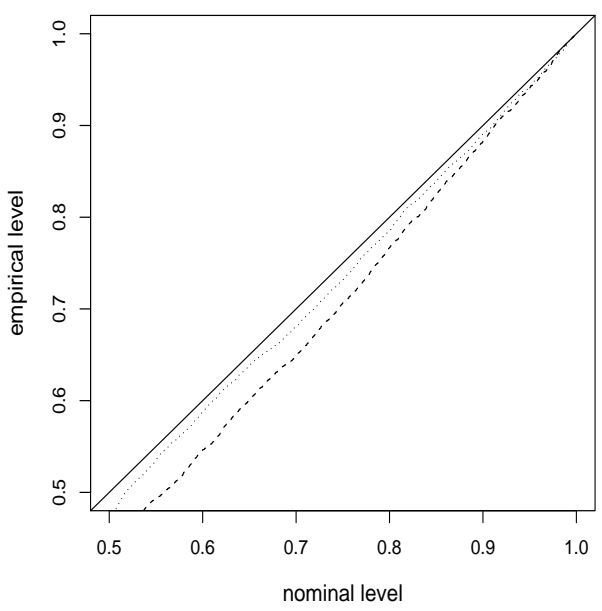

Figure 4: PP Plot of distribution of $T_{n}$ for $n=100$ (dashed line) and $n=$ 500 (dotted line) for hypothesis $H_{1,3}$ : $\alpha_{11}=0$ in case of estimated $\lambda$ 's.

$\pi_{2}=0.5$. The parameters of the state-dependent distributions were specified with $\lambda_{1}=2$, $\lambda_{2}=5$ and $\lambda_{3}=11$. The asymptotic distribution of the likelihood ratio statistic $T_{n}$ on the boundary of the hypothesis $H_{1,3}$ is (3).

Firstly, we consider the described model for fixed and known values of the $\lambda$ 's. Secondly, the $\lambda$ 's are considered as unknown parameters one has to estimate. For both cases we generate $N=5000$ samples of sizes $n=100,500$. The results are displayed in Figs. 7-8 using PP-plots. For fixed $\lambda$ 's the approximation is quite satisfactory even for small sample sizes $(n=100)$. Naturally, estimation of the $\lambda$ 's increases variation of the model. But for large sample sizes $(n=500)$ the approximation is quite good in this case, too.

\subsection{Series of epileptic seizure counts}

Albert (1991) proposed the use of two-state Poisson HMMs for series of daily seizure counts of epileptics. Using the implementation of the EM algorithm as suggested in Baum et al. (1970), Le et al. (1992) fit such models to the series of daily counts of epileptic seizures in one patient participating in a clinical trail at British Columbia's Children's Hospital. The originally published series consists of 225 observations, however, as indicated in MacDonald \& Zucchini (1997, p. 147), observations 92-112 should be deleted, thus we use the corrected data set of 204 observations.

In the neurology literature, Hopkins et al. (1985) proposed that the variation of seizure occurrences and its dependency structure could be modeled by a Markov chain. This is incorporated naturally in the two-state Poisson HMM, where the two states of the chain represent two states of seizure susceptibility. Haut (2006) pointed out that such Markovian dependence of seizure susceptibility allows estimates for the expected incidence of subsequent 
seizure days, which might be useful for recognition of seizure clusters.

A relevant question in this model is whether seizures actually occur in both states of the HMM, or whether there is only a single "seizure state", whereas, in the other state, no seizures occur. For the above mentioned data set MacDonald \& Zucchini (1997) fitted a stationary two-state Poisson HMM with estimated transition matrix

$$
\left(\begin{array}{ll}
0.973 & 0.027 \\
0.035 & 0.965
\end{array}\right)
$$

and seizure frequencies $\lambda_{1}=0.262$ and $\lambda_{2}=1.167$. Thus, we intend to test whether a model with $\lambda_{1}=0$ could be used instead, and therefore propose to test $H: \lambda_{1}=0$ as described in Example 2. Here, the asymptotic distribution of the likelihood ratio statistic $T_{n}$ under the hypothesis $H$ follows (3). However, the likelihood ratio test yields a value of $T_{n}=10.25$, which corresponds to a p-value of nearly 0 . Hence, the hypothesis $H$ is rejected, and seizures occur in both states of the HMM.

The estimate of the transition matrix yields for the stationary distribution $\hat{\pi}=(0.567,0.433)$. Therefore, the estimate indicates that state 1 with low seizure susceptibility is on average more frequently visited than state 2 . In order to test whether this observation is statistically significant, we test whether the hypothesis $H: \pi_{2} \geq \pi_{1}$ can be rejected. Again, the asymptotic distribution of the likelihood ratio statistic $T_{n}$ on the boundary of the hypothesis is (3). For this test the likelihood ratio statistic is $T_{n}=0.111$ with corresponding p-value 0.369 . Hence, we cannot reject the hypothesis $H$ at a $5 \%$-level, thus, there is not enough evidence that state 1 is more often visited than state 2 .

\section{Acknowledgements}

Jörn Dannemann acknowledges financial support from the Georg Lichtenberg program "Applied Statistics \& Empirical Methods". Hajo Holzmann gratefully acknowledges financial support from the Deutsche Forschungsgemeinschaft, grant MU 1230/8-2. The authors would like to thank Axel Munk and Walter Zucchini as well as the editor, the associate editor and two referees, for helpful comments.

\section{References}

Albert, P. S. (1991). A two-state Markov mixture model for time series of epileptic seizure counts. Biometrics 47, 1371-1381.

Arribas-Gil, A., Gassiat, E. \& Matias, C. (2006). Parameter Estimation in Pair-hidden Markov Models. Scand. J. Statist. 33, 651-671.

Bartolucci, F. (2006). Likelihood inference for a class of latent Markov models under linear 
hypothesis on the transition probabilities. J. R. Stat. Soc. Ser. B Stat. Methodol. 68, $155-178$.

Baum, L. E., Petrie, T., Soules, G. \& Weiss, N. (1970). A maximization technique occurring in the statistical analysis of probabilistic functions of Markov chains. Ann. Math. Statist. 41, 164-171.

Bickel, P. J., Ritov, Y. \& Rydén, T. (1998). Asymptotic normality of the maximum-likelihood estimator for general hidden Markov models, Ann. Statist. 26, 1614-1635.

Bickel, P. J., Ritov, Y. \& Rydén, T. (2002). Hidden Markov model likelihoods and their derivatives behave like i.i.d. ones. Ann. Inst. H. Poincaré Probab. Statist. 38, 825-846.

Cappé, O., Moulines, E. \& Rydén, T. (2005). Inference in hidden Markov models. Springer, New York.

Chernoff, H. (1954). On the distribution of the likelihood ratio. Ann. Math. Statist. 25, $573-578$.

Douc, R., Moulines, E. \& Rydén, T. (2004). Asymptotic properties of the maximum likelihood estimator in autoregressive models with Markov regime. Ann. Statist. 32, 2254-2304.

Giudici, P., Rydén, T. \& Vandekerkhove, P. (2000). Likelihood-ratio tests for hidden Markov models, Biometrics 56, 742-747.

Haut, S. R. (2006). Seizure clustering. Epilepsy \&3 Behavior 8, 50-55.

Holzmann, H., Munk, A., Suster, M. \& Zucchini, W. (2006). Hidden Markov models for circular and linear-circular time series, Environ. Ecol. Stat. 13, 325-347.

Hopkins, A., Davies, P. \& Dobson, C. (1985). Mathematical models of patterns of seizures: their use in the evaluation of drugs. Archives of Neurology 42, 463-467.

Le, N. D., Leroux, B. G. \& Puterman, M. L. (1992). Exact Likelihood Evaluation in a Markov Mixture Model for Time Series of Seizure Counts. Biometrics 48, 317-323.

Leroux, B. G. (1992). Maximum-likelihood estimation for hidden Markov models, Stochastic Process. Appl. 40, 127-143.

Leroux, B. G. \& Puterman, M. L. (1992). Maximum-penalized-likelihood estimation for independent and Markov-dependent mixture models. Biometrics 48, 545-558.

Lystig, T. C. \& Hughes, James P. (2002). Exact computation of the observed information matrix for hidden Markov models. J. Comput. Graph. Statist. 11, 678-689.

MacDonald, I. L. \& Zucchini, W. (1997). Hidden Markov and other models for discrete-valued time series. Chapman \& Hall, London.

Mardia, K. V. \& Sutton, T. W. (1975). On the modes of a mixture of two von Mises distri- 
butions. Biometrika 62, 699-701.

Rabiner, L. R. (1989). A tutorial on hidden Markov models and selected applications in speech recognition. Proceedings of the IEEE 77, 257-286.

Ray, S. \& Lindsay, B. G. (2005). The topography of multivariate normal mixtures. Ann. Statist. 33, 2042-2065.

Robertson, C. A. \& Fryer, J. G. (1969). Some descriptive properties of normal mixtures. Skandinavisk Aktuarietidskrift 52, 137-146.

Rydén, T., Terasvirta, T. \& Asbrink, S. (1998). Stylized facts of daily return series and the hidden Markov model. J. Appl. Econometrics 13, 217-244.

Self, S. G. \& Liang, K.-Y. (1987). Asymptotic properties of maximum likelihood estimators and likelihood ratio tests under nonstandard conditions. J. Amer. Statist. Assoc. 82, 605610 .

Silvapulle, M. J. \& Sen, P. K. (2005). Constrained statistical inference. Wiley, Hoboken, NJ.

Van den Broek, J. (1995). Score test for zero inflation in a Poisson distribution. Biometrics 51, 738-743.

\section{Appendix}

Definition 1. A set $\Theta \subset \mathbb{R}^{d}$ is said to be approximated at $\vartheta_{0}$ by a cone with vertex at $\vartheta_{0}$, denoted by $C_{\Theta, \vartheta_{0}}=C_{\Theta}$, if $\inf _{z \in C_{\Theta}}\|z-y\|=o\left(\left\|y-\vartheta_{0}\right\|\right)$ for all $y \in \Theta$ and if $\inf _{y \in \Theta}\|z-y\|=o\left(\left\|z-\vartheta_{0}\right\|\right)$ for all $z \in C_{\Theta}$.

A cone $C$ with vertex at $\vartheta_{0}$ is such that if $z \in C$, we also have $a\left(z-\vartheta_{0}\right)+\vartheta_{0} \in C$ for all $a>0$.

Assumption 1. We have $\vartheta_{0} \in \Theta$, and the MLE over $\Theta$ is strongly consistent.

Proof of (6). Set $a=1, b=2$. From (10.29), p. 362 in Cappé et al. (2005), we have to compute the asymptotic covariance matrix of the score vector

$$
E_{0}\left(\nabla_{\vartheta} \log \left(\pi_{X_{1}}(\vartheta)\right) \mid Y_{1: n}\right)+\sum_{k=1}^{n-1} E_{0}\left(\nabla_{\vartheta} \log \left(\alpha_{X_{k}, X_{k+1}}(\vartheta) \mid Y_{1: n}\right),\right.
$$

where $Y_{1: n}=\left(Y_{1}, \ldots, Y_{n}\right)$. Let $\alpha_{12}=\alpha$ and $\alpha_{21}=\beta$, so that $\vartheta=(\alpha, \beta)$. Neglect the first term in (10) for the moment. The derivative $\partial / \partial \alpha(\partial / \partial \beta$ is similar) of the second term are computed as

$$
\frac{\partial}{\partial \alpha} \log \left(\alpha_{x, x^{\prime}}(\vartheta)\right)=\frac{1}{\alpha} \delta_{(1,2)}\left(x, x^{\prime}\right)-\frac{1}{1-\alpha} \delta_{(1,1)}\left(x, x^{\prime}\right),
$$

so that the relevant sum in (10) (the derivative w.r.t. $\alpha$ in the second term) is

$$
\sum_{k=1}^{n-1} E_{0}\left(\frac{1}{\alpha} \delta_{(1,2)}\left(X_{k}, X_{k+1}\right)-\frac{1}{1-\alpha} \delta_{(1,1)}\left(X_{k}, X_{k+1}\right) \mid Y_{1: n}\right),
$$


where $\delta_{x}(y)=1$ if $x=y$ and $=0$ otherwise. The key observation is that $\alpha_{0}=\beta_{0}=1 / 2$, the $\left(X_{k}\right)$ are independent Bernoulli $1 / 2$ distributed with success probability $1 / 2$, and the $\left(Y_{k}\right)$ are also i.i.d. with a two-component mixture distribution. Using

$$
\delta_{(i, j)}\left(X_{k}, X_{k+1}\right)=\delta_{i}\left(X_{k}\right) \delta_{j}\left(X_{k+1}\right), \quad i, j \in\{1,2\},
$$

(11) can be rearranged as

$$
2 \sum_{k=1}^{n-1} E_{0}\left(\delta_{1}\left(X_{k}\right) \mid Y_{k}\right)\left(E_{0}\left(\delta_{2}\left(X_{k+1}\right) \mid Y_{k+1}\right)-E_{0}\left(\delta_{1}\left(X_{k+1}\right) \mid Y_{k+1}\right)\right),
$$

and a similar expression can be obtained for $\partial / \partial \beta$. Now

$$
E_{0}\left(\delta_{i}\left(X_{k}\right) \mid Y_{k}\right)=\frac{f\left(Y_{k} ; \theta_{i}\right)}{f\left(Y_{k} ; \theta_{1}\right)+f\left(Y_{k} ; \theta_{2}\right)}, \quad i \in\{1,2\},
$$

Introducing the random variables

$$
\begin{aligned}
Z_{k, 1} & =\frac{f\left(Y_{k} ; \theta_{1}\right)}{f\left(Y_{k} ; \theta_{1}\right)+f\left(Y_{k} ; \theta_{2}\right)} \frac{f\left(Y_{k+1} ; \theta_{2}\right)-f\left(Y_{k+1} ; \theta_{1}\right)}{f\left(Y_{k+1} ; \theta_{1}\right)+f\left(Y_{k+1} ; \theta_{2}\right)}, \\
Z_{k, 2} & =\frac{f\left(Y_{k} ; \theta_{2}\right)}{f\left(Y_{k} ; \theta_{1}\right)+f\left(Y_{k} ; \theta_{2}\right)} \frac{f\left(Y_{k+1} ; \theta_{1}\right)-f\left(Y_{k+1} ; \theta_{2}\right)}{f\left(Y_{k+1} ; \theta_{1}\right)+f\left(Y_{k+1} ; \theta_{2}\right)}
\end{aligned}
$$

the components of the score are given by $S_{n, 1}=2 \sum_{k=1}^{n-1} Z_{k, 1}, S_{n, 2}=2 \sum_{k=1}^{n-1} Z_{k, 2}$. Although the $Z_{k, 1}$ 's are not i.i.d., the covariances for different $k$ 's are 0 , and the correlation can be computed as

$$
\rho=\frac{E_{0} Z_{k, 1} Z_{k, 2}}{\left(E_{0} Z_{k, 1}^{2} E_{0} Z_{k, 2}^{2}\right)^{1 / 2}} .
$$

For the first term in (10), one has that $E_{0}\left(\nabla_{\vartheta} \log \left(\pi_{X_{1}}(\vartheta)\right) \mid Y_{1: n}\right)=E_{0}\left(\nabla_{\vartheta} \log \left(\pi_{X_{1}}(\vartheta)\right) \mid Y_{1}\right)$, of which the contribution to the asymptotic covariance matrix is zero. This proves (12), and (6) follows from case 7 in Self \& Liang (1987). Note that this example generalizes the Example 4.3 in Bickel et al. (2002), who consider reversible Markov chains and thus only have a single parameter.

Proof of (8). We use (2) and a coordinate transformation to obtain

$$
\begin{aligned}
T_{n} \stackrel{\mathcal{L}}{\rightarrow} T & :=\min _{z \in H}(Z-z)^{T}(Z-z)-\min _{z \in H \cup K}(Z-z)^{T}(Z-z) \\
& =\left\|Z-Z_{H}\right\|^{2}-\min \left(\left\|Z-Z_{H}\right\|^{2},\left\|Z-Z_{K}\right\|^{2}\right)
\end{aligned}
$$

where $Z$ is a bivariate standard normal random variable, $H=\mathcal{J}_{0}^{1 / 2}\left(\mathbb{R}^{-} \times \mathbb{R}^{-}\right)$and $K=\mathcal{J}_{0}^{1 / 2}\left(\mathbb{R}^{+} \times\right.$ $\mathbb{R}^{+}$), as shown in Figure $5, Z_{H}$ and $Z_{K}$ denote the orthogonal projections of $Z$ onto $H$ and $K$ respectively, and $\phi=\cos ^{-1} \rho$. Firstly, we calculate the distribution of $T$ conditional on the event $\{Z \in$ region 1 or $1 a\}$ and the weight $P(Z \in$ region 1 or $1 a)$. If $Z$ is in region 1 , then obviously both terms in (13) are zero. For region $1 a$ the difference is zero as well, since $\left\|Z-Z_{H}\right\| \leq\left\|Z-Z_{K}\right\|$. Hence the conditional distribution of $T$ in region 1 and $1 a$ is $\chi_{0}^{2}$. As displayed in the figure its weight is $\frac{1}{2 \pi}\left(\phi+2 \frac{\pi-\phi}{2}\right)=\frac{1}{2}$.

If $Z$ is in region 2 and therefore in $K$ the term $\left\|Z-Z_{K}\right\|$ is zero. Hence the distribution of $T$ conditional on $\{Z \in$ region 2$\}$ is determined by the first term, which gives a $\chi_{2}^{2}$ - distribution, since $Z_{H}=0$. The weight is given by $\frac{\pi-\phi}{2 \pi}$. Observe that $Z$ is determined by its argument $\psi=\arg (Z)$ and its length $r(Z)$ 


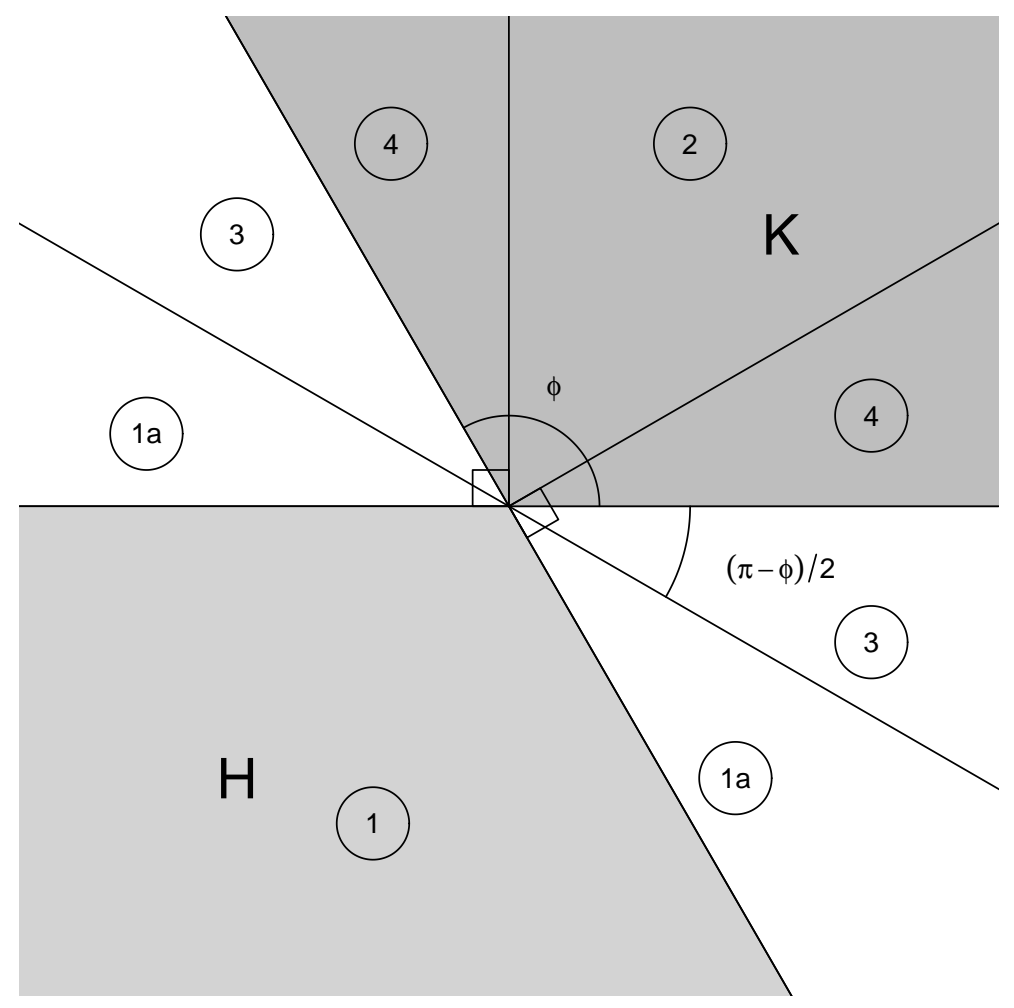

Figure 5: Diagram of the parameter space of Example 3.

by $Z=(r \cos \psi, r \sin \psi)$, where $r^{2}$ is $\chi_{2}^{2}$-distributed and $\psi$ is uniformly distributed on $[0,2 \pi)$, and $r^{2}$ and $\psi$ are independent. If $Z$ is now in region 3, i.e. $\psi \in[\phi,(\phi+\pi) / 2) \cup[-(\pi-\phi) / 2,0)$, one has $\left\|Z-Z_{H}\right\|^{2}=r^{2} \sin ^{2}(\pi-\psi)$ and $\left\|Z-Z_{K}\right\|^{2}=r^{2} \sin ^{2}(\psi-\phi)$. Setting

$$
a_{1}(\psi, \phi)=\sin ^{2}(\pi-\psi)-\sin ^{2}(\psi-\phi),
$$

for fixed $\psi$ the difference $T(\psi)=\left\|Z-Z_{H}\right\|^{2}-\left\|Z-Z_{K}\right\|^{2}=r^{2} a_{1}(\psi, \phi)$ has a rescaled $\chi_{2}^{2}$-distribution with density $p(t ; \phi, \psi)=\frac{1}{2 a_{1}(\psi, \phi)} \exp \left(-\frac{t}{2 a_{1}(\psi, \phi)}\right)$. Hence the density of $T$ is an averaged rescaled $\chi_{2}^{2}$-distribution $P_{1}(\phi)$ with density

$$
h_{1}(t ; \phi)=\frac{2}{\pi-\phi} \int_{\phi}^{(\phi+\pi) / 2} \frac{1}{2 a_{1}(\psi, \phi)} \exp \left(-\frac{t}{2 a_{1}(\psi, \phi)}\right) d \psi
$$

The weight of region 3 is given by $\frac{\pi-\phi}{2 \pi}$.

For region 4 one proceeds similarly. For $\psi \in[0, \phi-\pi / 2) \cup[\pi / 2, \phi)$ one has $\left\|Z-Z_{H}\right\|^{2}=r^{2} \cos ^{2} \psi$ and trivially $\left\|Z-Z_{K}\right\|=0$. Setting

$$
a_{2}(\psi)=\cos ^{2}\left(\psi-\frac{\pi}{2}\right),
$$

this yields a conditional distribution $P_{2}(\phi)$ of $T$ with density

$$
h_{2}(t ; \phi)=\frac{2}{2 \phi-\pi} \int_{\pi / 2}^{\phi} \frac{1}{2 a_{2}(\psi)} \exp \left(-\frac{t}{2 a_{2}(\psi)}\right) d \psi
$$

with weight $2 \frac{\phi-\frac{\pi}{2}}{2 \pi}=\frac{2 \phi-\pi}{2 \pi}$. 